\title{
ESTIMATION OF MICROMIXING PARAMETERS FROM TRACER CONCENTRATION FLUCTUATION MEASUREMENTS
}

\author{
MICHAEL L. CALL and ROBERT H. KADLEC \\ Department of Chemical Engineering, University of Michigan, Ann Arbor, MI 48109-2136, IJ.S.A.
}

(Received 14 March 1988; accepted 20 October 1988)

\begin{abstract}
A method of micromixing parameter estimation is proposed which is convenient and applicable to a wide variety of models. One conducts a typical tracer response test, except that in addition to the (timeaveraged) mean tracer response, the variance of the tracer response is also recorded as a function of time. Micromixing parameters for a given model are determined by equating the predicted and measured values of the time response of the tracer concentration variance. The predicted tracer concentration fluctuation response has been determined for both step and pulse tracer tests, for the IEM model, the coalescenceredispersion model, and the two-, three- and four-environment models. Parameter estimates were calculated by minimizing the mismatch between predicted and measured concentration fluctuation responses. Preliminary numerical results indicate that the method provides satisfactory parameter estimates, even from moderately noisy measurements. These results also show that statistical analysis of the fit to measured response data can provide discrimination between competing mixing models.
\end{abstract}

\section{INTRODUCTION}

Much research has been devoted to developing methods for predicting the behavior of imperfectlymixed chemical reactors. This research has resulted in a number of mathematical models for predicting the conversion behavior of imperfectly-mixed reactors. Unfortunately, these models are not well utilized in industrial practice. One reason is the difficulty of estimating mixing parameters. Comparison between model and reactor test data is a reliable and widely applicable method, but is often costly, impractical, or computationally intensive. The use of a "turbulence analogy" provides crude parameter estimates with little effort, but this method is applicable to a limited number of models. A method of parameter estimation is proposed in this paper which is convenient, widely applicablè, and computationally efficient.

The method is based upon dynamic tracer response testing, and thus requires no effort beyond that required to determine the residence time distribution alone. Experimentally, one conducts a routine tracer response test, except that the tracer concentration fluctuation response is recorded as a function of time, in addition to (and simultaneously with) the mean tracer concentration response. The mixing parameter(s) for a model can then be determined by choosing value(s) to minimize the mismatch between the measured tracer concentration fluctuation response and that predicted by the model.

If one conducted a tracer test and observed the reactor exit at time $t$, one would observe many concentrations, due to the many turbulent eddies which comprise the effluent. If this tracer test were repeated, a different set of concentrations would be observed, due to the random nature of the turbulence in the reactor. If the tracer test were repeated ad infinitum, these observed concentrations would form some distribution, with a definite mean and variance. By definition, the mean tracer concentration at time $t$ is the mean of this distribution, and the tracer concentration fluctuation at time $t$ is the variance of this distribution. When applied for all times, $t$, this defines the tracer concentration mean response and the tracer concentration fluctuation response.

To measure the tracer concentration mean response, it is sufficient to use a low-spatial-resolution measurement probe, with a moderately slow response time. To measure the tracer concentration fluctuation response, one would ideally sample the concentration at all points across the exit plane and calculate their variance. This, of course, is not possible. Instead, one must sample a point (single-eddy) concentration, and use the variance of samples at several consecutive times to approximate the concentration fluctuation. To make such measurements requires the use of highspatial-resolution measurement probes with fast response times.

McKelvey et al. (1975) used a light probe to measure concentration fluctuations by colorimetry. They found that noise and lack of spatial resolution were serious problems, and recommended that conductimetry be used in future work. Bennani et al: (1985) measured concentration fluctuations with a conductimetric microprobe developed by Gibson and Schwarz (1963) having a spatial resolution of $2500 \mu \mathrm{m}$. Calculations showed that some of their measurements were significantly in error, presumably due to the low spatial resolution. Barthole et al. (1982) made similar measurements with a microprobe having a spatial resolution of $200 \mu \mathrm{m}$ and a response time of $5 \mathrm{~ms}$. They also concluded that the spatial resolution of their probe was inadequate. More recently, Hippler et al. (1985) have developed a conductimetric microprobe 
having a spatial resolution of about $40 \mu \mathrm{m}$ with a response time of about $3 \mathrm{~ms}$

Hanley and Mischke (1978) and Hippler et al. (1985) used conductimetric microprobes to measure the $\mathrm{dy}$ namic tracer response at the outlet of a reactor, and were thus able to determine concentration fluctuations and the residence time distribution simultaneously. This approach has the advantage that information about micromixing is obtained with no experimental effort beyond that required to obtain information about macromixing alone.

The concept of using tracer concentration fluctuations to estimate mixing parameters is not new. Kattan and Adler (1967) mentioned the possibility of utilizing tracer concentration fluctuations to determine the mixing function of their imperfectly-mixed plug flow reactor model. Evangelista et al. (1969) demonstrated how the tracer concentration fluctuation response can be used to estimate the mixing parameter of the imperfectly-mixed CSTR model of Curl (1963). Villermaux and Devillon (1972) and Zwietering (1984) both show how tracer concentration fluctuation responses can be used to estimatc the parameters of their imperfectly-mixed CSTR models.

None of those papers considers the use of tracer concentration fluctuation responses for parameter estimation with mixing models applicable to arbitrary residence time distributions. This case is considered by Hanley and Mischke (1978) and Hippler et al. (1985). However, they use an integral measure based on the tracer concentration fluctuation response, and they use it purely as a correlative parameter, arriving at no theoretical relationship between mixing parameters and the tracer concentration fluctuation response. The approach of the present paper differs from that of the papers cited above in that predicted tracer concentration fluctuation responses are determined for mixing models applicable to reactors of arbitrary residence time distribution, and in that the entire tracer concentration fluctuation response is utilized in the parameter estimation process.

\section{MIXING MODEIS}

A great number of models have been developed to model the influence of imperfect mixing in chemical reactors. ${ }^{+}$All of these models consider the multiplicity of concentrations present in the reactor. Consequently, all predict a distribution of concentrations in the reactor effluent, as is observed in reality. Lagrangian mixing models may be broadly classified, according to how they handle the approximation of the distribution of concentrations, as:

(1) environment models--where material entering the reactor encounters different regions, each with either no mixing or complete mixing;

(2) diffusion-interaction models- where material entering the reactor uniformly encounters some

\footnotetext{
TFor comprehensive reviews of this subject see, for example, Ritchie and Tobgy (1978) or Villermaux (1983).
}

intermediate level of mixing; or

(3) composite models - where material entering the reactor encounters different regions, each with some characteristic intermediate level of mixing.

\section{Environment models}

The environment models conceptually divide the reactor into a number of regions, referred to as environments, each of which is in a state of either complete segregation or maximum mixedness. Reactants enter one of the environments, travel through one or more of the environments in the reactor, and eventually leave the reactor. Various models differ principally in the number of environments and in the distribution of material between the environments.

The first such model was developed by $\mathrm{Ng}$ and Rippin (1965). In this model, material enters into a segregated environment corresponding to the periphery of the reactor, subsequently transfers to a maximally-mixed environment corresponding to the impeller region of the reactor, and exits the reactor from both environments. The rate of transfer of any matcrial from the entcring (scgregated) environment to the leaving (maximally-mixed) environment is assumed to be proportional to the amount of the material remaining in the entering environment. This assumption roughly corresponds to quasi-random turbulent transfer. By varying the transfer constant, $R$, from zero to infinity, the mixing state is systematically varied from complete segregation to maximum mixedness.

Several other environment models have been developed for premixed feed reactors, including the models proposed by Weinstein and Adler (1967), Villermaux and Zoulalian (1969), Methot and Roy (1971), and Valderrama and Gordon (1979). Nishimura and Matsubara (1970) developed a generalized two-environment model, in which the distribution of material between the entering and leaving environments is determined by the segregation function $s(\alpha, \lambda)$. It has been shown that, with a suitably chosen segregation function, all of the above models are special cases of the generalized two-environment model.

Ritchie and Tobgy (1979) developed a three-environment model for unmixed feed reactors. Their model was similar to the two-environment model of $\mathrm{Ng}$ and Rippin (1965), except that it provided a separate (segregated) entering environment for each feed with subsequent transfer to a single (maximallymixed) leaving environment. Mehta and Tarbell (1983) later developed a four-environment model. Their model is similar to the three-environment model, except that material from each entering environment transfers to a separate leaving environment. The mixing of material from different feed streams is provided for by the direct transfer of material between the two leaving environments.

\section{Diffusion-interaction models}

Diffusion-interaction models differ from environment models in that some intermediate degree of 
mixing is presumed to exist throughout the reactor. Rather than considering the (uniform) concentrations in each of a small number of environments, the distribution of concentrations within the reactor is addressed directly. Diffusion-interaction models can be divided into two categories, based on their presumed mixing mechanisms: (1) coalescence-redispersion (CRD) models, and (2) IEM models. Both of these types of models are discussed in more detail below.

$C R D$ models. CRD models envision the reacting fluid as being composed of a large number of small packets of fluid. The packets enter the reactor, react, mix with other packets, and eventually exit the reactor. The mixing process is modeled as the joining (coalescence) of two packets, the complete mixing of their contents, and the immediate breaking apart (redispersion) of the two packets. By varying the rate at which these mixing events occur, the state of mixing in the reactor can be systematically varied from complete segregation to maximum mixedness.

The first CRD model was developed by Curl (1963) for dispersed-phase mixing in a CSTR. Using population balance techniques, Curl derived the unsteadystate equation of change for the concentration distribution, $p(C, t)$, in terms of a partial integro-differential equation.

Unfortunately, the partial integro-differential equation can be solved exactly only for the case of a firstorder reaction, where the degree of mixing does not affect the mean conversion. Further, extension of the model to multiple reactions gives rise to multiple integrals in the mixing term, making even numerical solution of the equation untenable. As a result, Spielman and Levenspiel (1965) developed a stochastic method for solving Curl's CRD model based on direct simulation of the flow and mixing events for a representative number of packets. Kattan and Adler (1967) developed a stochastic CRD model for unmixed feed plug flow reactors, using a coalescence rate which varied down the length of the reactor.

The CRD modeling of reactors of arbitrary RTD is a more difficult problem than that for CSTRs and PFRs, since it is not obvious how to choose the packets for mixing and exiting the reactor so as to attain the desired RTD. This problem was solved by Kattan and Adler (1972) by modelling the reactor as a PFR with side inlets (as in the maximum-mixedness model), but with incomplete radial mixing occurring by a CRD mechanism. They not only derived the unsteady-state equation of change for the concentration distribution $p(C, \lambda, t)$ but also presented a stochastic form of the model which was more suitable for numerical solution.

Treleaven and Tobgy (1972) developed a stochastic CRD model for reactors having multiple feed streams with distinct RTDs. Their model is similar to that of Kattan and Adler (1972), except that there are separate source terms for each feed stream in the mass balance.
IEM models. IEM models are conceptually similar to CRD models, in that they hoth envision molecular mixing as the result of an exchange of material between distinct packets of material in the reactor. In the IEM description of mixing, however, it is assumed that each packet undergoes a great many such collisions, each of which results in only a small amount of material exchanged. The combined effect of these collisions is as if the packet had exchanged part of its original contents for material having the mean concentration of all the packets. This is the source of the name Interaction by Exchange with the Mean.

This model was developed for the CSTR independently by Villermaux and Devillon (1972) and Costa and Trevissoi (1972). Villermaux (1983) also utilized the IEM mixing mechanism to derive models for single and multiple feed stream reactors of arbitrary RTD, analogous to the CRD models of Kattan and Adler (1972) and Treleaven and Tobgy (1972).

\section{Composite models}

Composite models combine aspects of both environment models and diffusion-interaction models to obtain more complex descriptions of the mixing process. They may be thought of as generalizations of environment models, where some intermediate degree of mixing is provided within each environment.

Goto and Matsubara (1975) proposed a composite model based on the generalized two-environment model of Nishimura and Matsubara (1970). In this new model, mixing occurs in each environment by a CRD mechanism. In the entering environment, mixing occurs among packets of equal age, while in the leaving environment, mixing occurs among packets of equal residual lifetime. Ritchie (1980) developed a similar composite model applicable to reactors having separate feed streams with distinct residence time distributions.

Klein et al. (1980) developed a model for an ideallymacromixed reactor. Like the "shrinking aggregate" model of Plasari et al. (1978) the reacting fluid is considered as a collection of large and small aggregates. The material in the entering environment (large aggregates) is transferred to the leaving environment (small aggregates) by the erosion of the large aggregates. Klein et al. (1980) assumed that the material in the leaving environment was not maximally mixed, but instead experienced an intermediate degree of mixing, provided by an IEM mixing mechanism.

\section{The master model}

Much of the analysis that follows is based on a single "master" model which assumes the form of several previously proposed models as special cases. In this master model, fluid from each of two feed streams enters into a single entering environment, subsequently transfers to a single leaving environment, and exits the reactor from both the entering and the leaving environment. The transfer between the two environments is governed by an environment funetion, $s(\alpha, \lambda)$, such that the fraction of material of age $\alpha$ 
and residual lifetime $\lambda$ which remains in the entering environment is $s(\alpha, \lambda)$. In the entering environment, mixing takes place among material having a common age and common residual lifetime, at a rate which is a function of age. In the leaving environment, mixing takes place among material having a common residual lifetime, at a rate which is a function of residual lifetime. Mixing may be assumed to take place by either a CRD mechanism or an exchange-with-themean mechanism.

The equations of change describing concentration distributions in the master model with mixing by a CRD mechanism are:

$$
\begin{aligned}
& \frac{\partial}{\partial \xi}\left[P_{b}(\vec{C}, \xi)\right]=-\sum_{j=1}^{m} \frac{\partial}{\partial C}-\left[\mathscr{R}_{j}(\vec{C}) P_{b}(\vec{C}, \xi)\right] \\
& +I_{E}(\xi)\left[2^{m} \int_{\gamma_{1}=-\infty}^{\gamma_{1}=\infty} \cdots \int_{\gamma_{m}=-\infty}^{\gamma_{m}=\infty} P_{b}(\vec{C}+\vec{\gamma}, \xi) P_{b}(\vec{C}\right. \\
& \left.-\vec{\gamma}, \xi) \mathrm{d} \vec{\gamma}-P_{b}(\vec{C})\right] \\
& P_{b}(\vec{C}, \xi=0)=\sum_{j=1}^{2} \phi_{j} \frac{f_{j}(\alpha+\lambda)}{f(\alpha+\lambda)} P_{f, j}(\vec{C}, t-\alpha) \\
& P_{E}(\vec{C}, \alpha, \lambda, t)=P_{b}(\vec{C} ; \xi=\alpha)
\end{aligned}
$$

for the entering environment concentration distribution,

$$
\begin{aligned}
& \frac{\partial}{\partial t}\left[\operatorname{II}(\lambda) P_{L}(\dot{C}, \lambda, t)\right]=\frac{\partial}{\partial \lambda}\left[\operatorname{II}(\lambda) P_{L}(\vec{C}, \lambda, t)\right] \\
& \quad+\int_{0}^{\infty} P_{E}(\vec{C}, \alpha, \lambda, t) f(\alpha+\lambda)\left(\frac{\partial s}{\partial \lambda}-\frac{\partial s}{\partial \alpha}\right) \mathrm{d} \alpha \\
& \quad-\operatorname{II}(\lambda) \sum_{j=1}^{m} \frac{\partial}{\partial C_{j}}\left[\mathscr{R}_{j}(\vec{C}) P_{L}(\vec{C}, \lambda, t)\right] \\
& \quad+I_{L}(\lambda) \operatorname{II}(\lambda)\left[2^{m} \int_{\gamma_{1}=-\infty}^{\gamma_{1}=\infty} \ldots \int_{\gamma_{m}}^{\gamma_{m}=\infty} P_{L}(\vec{C}\right. \\
& \left.\quad+\vec{\gamma}, \lambda, t) P_{L}(\vec{C}-\vec{\gamma}, \lambda, t) \mathrm{d} \vec{\gamma}-P_{L}(\vec{C}, \lambda, t)\right]
\end{aligned}
$$

for the leaving environment concentration distribution, and

$$
\begin{aligned}
P_{x}(\vec{C}, t) & =\operatorname{II}(\hat{\lambda}=0) P_{L}(\vec{C}, \lambda=0, t) \\
& +\int_{0}^{\infty} P_{E}(\vec{C}, \alpha, \lambda=0, t) f(\alpha) s(\alpha, \lambda=0) \mathrm{d} \alpha
\end{aligned}
$$

for the reactor effluent concentration distribution, where

$$
\mathbf{I I}(\lambda)=\int_{0}^{\infty} f(\alpha+\lambda)[1-s(\alpha, \lambda)] \mathrm{d} \alpha .
$$

Call (1989) has used population balance techniques to show that the equations of change describing the master model with mixing by an exchange-with-themean mechanism are

$$
\frac{\partial}{\partial \xi}\left[P_{b}(\vec{C}, \xi)\right]=-\sum_{j=1}^{m} \frac{\partial}{\partial C_{j}}\left[\mathscr{R}(\vec{C}) P_{b}(\vec{C}, \xi)\right]
$$

$$
\begin{gathered}
-\beta_{E}(\xi) \sum_{j=1}^{m} \frac{\partial}{\partial C_{j}}\left[\left(M_{1, b, j}(\xi)-C_{j}\right) P_{b}(\vec{C}, \xi)\right] \\
P_{b}(\vec{C}, \xi=0)=\sum_{j=1}^{2} \phi_{i} \frac{f_{j}(\alpha+\lambda)}{f(\alpha+\lambda)} P_{f, j}(\vec{C}, t-\alpha) \\
P_{E}(\vec{C}, \alpha, \lambda, t)=P_{b}(\vec{C}, \xi=\alpha)
\end{gathered}
$$

for the entering environment concentration distribution,

$$
\begin{aligned}
& \frac{\partial}{\partial t}\left[\operatorname{II}(\lambda) P_{L}(\vec{C}, \lambda, t)\right]=\frac{\partial}{\partial \lambda}\left[\operatorname{II}(\lambda) P_{L}(\vec{C}, \lambda, t)\right] \\
& \quad+\int_{0}^{\infty} P_{E}(\vec{C}, x, \lambda, t) f(\alpha+\lambda)\left(\frac{\partial s}{\partial \lambda}-\frac{\partial s}{\partial x}\right) \mathrm{d} \alpha \\
& \quad-\operatorname{II}(\lambda) \sum_{j=1}^{m} \frac{\partial}{\partial C_{j}}\left[\mathscr{R}_{j}(\vec{C}) P_{L}(\vec{C}, \lambda, t)\right] \\
& \quad-\beta_{L}(\lambda) \operatorname{II}(\lambda) \sum_{j=1}^{m} \frac{\partial}{\partial C_{j}}\left[\left(M_{1, L . j}(\hat{\lambda}, t)-C_{j}\right) P_{L}(\vec{C}, \lambda, t)\right]
\end{aligned}
$$

for the leaving environment concentration distribution, and

$$
\begin{aligned}
P_{x}(\vec{C}, t)= & \operatorname{II}(\lambda=0) P_{L}(\vec{C}, \lambda=0, t) \\
& +\int_{0}^{\infty} P_{E}(\mathcal{C}, \alpha, \lambda=0, t) f(\alpha) s(\alpha, \lambda=0) \mathrm{d} \alpha
\end{aligned}
$$

for the reactor effluent concentration distribution.

The master model is not proposed as a practical model to be used directly in reactor simulation. It is clear that the effort required to estimate three distributed parameters could rarely (if ever) be justified, and that less complex models would provide adequate simulation results.

Rather, the master model is proposed as a conceptual tool for the analysis of mixing models. The master model assumes the form of 18 previously proposed reactor models as special cases, including all of the models discussed, except for the four-environment model of Mehta and Tarbell (1983). The relationships between these models and the master model are illustrated graphically in Fig. 1. An awareness of these relationships is beneficial in that it elucidates the assumptions (about the mixing patterns and mechanism) implicit in selecting a particular reactor mixing model.

\section{MODEL RESPONSES}

To determine the tracer concentration fluctuation response predicted by a model, that reactor model must be formulated for unsteady-state application. From such a formulation, equations of change can be determined for the first two moments of the effluent concentration distribution for a single, inert, component. Given appropriate initial conditions, these equations can be solved for moment responses, from which the tracer concentration fluctuation response follows. 


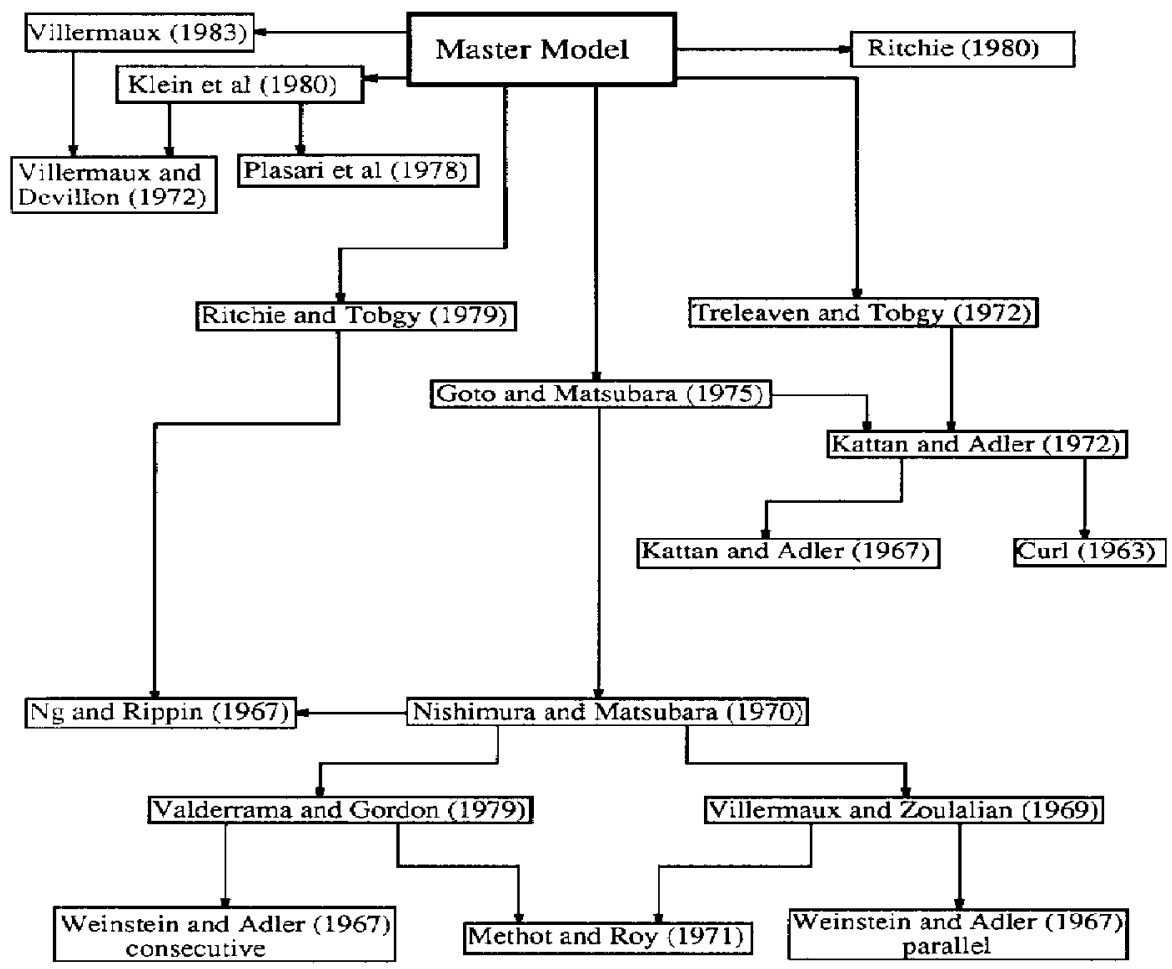

Fig. 1. Relationship between the master model and existing reactor models.

\section{Master model response}

For the master model with mixing by a CRD mochanism, the equations of change for the first two moments of the reactor effluent concentration distribution are:

$$
\begin{gathered}
\frac{\partial}{\partial \xi}\left[M_{1, b}(\xi)\right]=0 \\
M_{L, b}(\xi=0)=\sum_{j=1}^{2} \phi_{j} \frac{f_{j}(\alpha+\lambda)}{f(\alpha+\lambda)} M_{1, f, j}(t-\alpha)
\end{gathered}
$$

and

$$
M_{1, E}(\alpha, \lambda, t)=M_{1, b}(\xi=\alpha)
$$

for the mean concentration in the entering environment,

$$
\begin{aligned}
\frac{\partial}{\partial t}\left[\mathbf{I I}(\lambda) M_{1, L}(\lambda, t)\right]=\frac{\partial}{\partial \lambda}\left[\operatorname{II}(\lambda) M_{1, L}(\lambda, t)\right] \\
+\int_{0}^{\infty} M_{1, E}(\alpha, \lambda, t) f(\alpha+\lambda)\left(\frac{\partial s}{\partial \lambda}-\frac{\partial s}{\partial \alpha}\right) \mathbf{d} \alpha
\end{aligned}
$$

for the mean concentration in the leaving environment,

$$
\begin{aligned}
M_{1, x}(t)= & \Pi(\lambda=0) M_{1, L}(\lambda=0, t) \\
& +\int_{0}^{\infty} M_{1, E}(\alpha, \lambda=0, t) f(\alpha) s(\alpha, \lambda=0) \mathrm{d} \alpha
\end{aligned}
$$

for the mean concentration in the reactor effluent,

$$
\begin{aligned}
& \frac{\partial}{\partial \xi}\left[M_{2, b}(\xi)\right]=\frac{1}{2} I_{E}(\xi)\left[M_{1, b}^{2}(\xi)-M_{2, b}(\xi)\right] \\
& M_{2, b}(\xi=0)=\sum_{j=1}^{2} \phi_{j} \frac{f_{j}(\alpha+\lambda)}{f(\alpha+\lambda)} M_{2, s . j}(t-\alpha)
\end{aligned}
$$

and

$$
M_{2, E}(\alpha, \lambda, t)=M_{2, b}(\xi=\alpha)
$$

for the mean squared concentration in the entering environment,

$$
\begin{aligned}
& \frac{\partial}{\partial t}\left[\operatorname{II}(\lambda) M_{2, L}(\lambda, t)\right]=\frac{\partial}{\partial \lambda}\left[\operatorname{II}(\lambda) M_{2, L}(\lambda, t)\right] \\
& \quad+\int_{0}^{\infty} M_{2, s}(\alpha, \lambda, t) f(\alpha+\lambda)\left(\frac{\partial s}{\partial \lambda}-\frac{\partial s}{\partial \alpha}\right) \mathrm{d} \alpha \\
& \quad+\frac{1}{2} I_{L}(\lambda) \operatorname{II}(\lambda)\left[M_{1, L}^{2}(\lambda, t)-M_{2, L}(\lambda, t)\right]
\end{aligned}
$$

for the mean squared concentration in the leaving environment, and

$$
\begin{aligned}
M_{2, x}(t) & =\operatorname{II}(\lambda=0) M_{2, L}(\lambda=0, t) \\
& +\int_{0}^{\infty} M_{2, E}(\alpha, \lambda=0, t) f(\alpha) s(\alpha, \lambda=0) \mathrm{d} \alpha
\end{aligned}
$$

for the mean squared concentration in the reactor effluent.

Call (1989) has developed analogous equations for mixing by an exchangc-with-the-mean mechanism and has shown that the resulting equations are identical to eqs (12)-(21), provided that the correspondence 
$4 \beta_{E}(\alpha)=I_{E}(\alpha)$ and $4 \beta_{L}(\lambda)=I_{L}(\lambda)$ is maintained.

Before the moment response equations can be solved, the appropriate boundary conditions must be specified. In this paper, boundary conditions corresponding to step and finite pulse tracer tests are considered. In the step tracer test, it is assumed that no tracer is initially present in the reactor, and that for times $t \geqslant 0$, a fraction $q$ of the flow into inlet stream $i(i=1$ or 2$)$ is replaced by tracer of concentration $C_{f}$, while inlet stream $k$ remains undisturbed. For this situation the feed moments are described by

$$
M_{n, f, i}(t)=q C_{f}^{n} U(t) \text { and } M_{n, f, k}(t)-0 .
$$

In the finite pulse tracer test, it is assumed that no tracer is initially present in the reactor, and that for times $0 \leqslant t \leqslant \delta$, a fraction $q$ of the flow into inlet steam $i$ is replaced by tracer of concentration $C_{f}$, while inlet stream $k$ remains undisturbed. For this situation, the feed moments are described by

$M_{n_{-} f, i}(t)=q C_{f}^{n}[U(t)-U(t-\delta)]$ and $M_{n, f, k}(t)=0$.

The equations of change were solved by first converting the PDEs in eqs (15) and (20) to ODEs by a change in the independent variables from $t$ and $\lambda$ to $T=t+\lambda$ and $\lambda$. Equation (17) was solved by using the integrating factor method with an integrating factor of $K_{E}^{-1}(\alpha)$. Equation (15), after conversion to an ODE, was solved by direet integration, with the resulting double integral simplified by a change of independent variables from $\alpha$ and $\lambda$ to $\alpha$ and $\xi=\alpha+\lambda$. Equation (20), after conversion to an ODE, was solved by the integrating factor method with an integrating factor of $K_{L}(\lambda)$. These intermediate results were combined according to eq. (21) to yield the mean squared exit concentration, and the squared mean exit concentration was subtracted to give the tracer concentration fluctuation response.

For the step tracer input, this solution procedure gives the tracer concentration fluctuation response as

$$
\begin{aligned}
& \frac{\sigma^{2}(t)}{C_{f}^{2}}=q \phi_{i}\left[\int_{0}^{t} K_{E}(\alpha) f_{i}(\alpha) s(\alpha, \lambda=0) \mathrm{d} \alpha+\int_{\lambda=0}^{\lambda=t} K_{L}(\lambda)\right. \\
& \left.\times \int_{\alpha=0}^{\alpha=t-\lambda} K_{E}(\alpha) f_{i}(\alpha+\lambda)\left(\frac{\partial s}{\partial \lambda}-\frac{\partial s}{\partial \alpha}\right) \mathrm{d} \alpha \mathrm{d} \lambda\right] \\
& +q^{2} \phi_{i}^{2}\left\{-F_{i}^{2}(t)+\int_{\lambda=0}^{\lambda=1} K_{L}(\lambda) \int_{\alpha=0}^{\alpha=t-\lambda}\right. \\
& \times\left[1-K_{E}(\alpha)\right] \frac{f_{i}^{2}(\alpha+\lambda)}{f(\alpha+\lambda)}\left(\frac{\partial s}{\partial \lambda}-\frac{\partial s}{\partial \alpha}\right) \mathrm{d} \alpha \mathrm{d} \lambda \\
& +\int_{\alpha=0}^{\alpha=t}\left[1-K_{E}(\alpha)\right] \frac{f_{i}^{2}(\alpha)}{f(\alpha)} s(\alpha, \lambda=0) \mathrm{d} \alpha \\
& +\frac{1}{2} \int_{\lambda=0}^{\lambda=t} I_{L}(\lambda) K_{L}(\lambda) \\
& \quad\left[F_{i}(t)-F_{i}(\lambda)-\int_{\alpha=0}^{\alpha=t-\lambda} f(\alpha+\lambda) s(\alpha, \lambda) \mathrm{d} \alpha\right]^{2} \\
& \times
\end{aligned}
$$

where $K_{E}$ and $K_{L}$ are auxiliary mixing functions defined by

$$
\begin{aligned}
& K_{E}(\xi)=\exp \left[-\frac{1}{2} \int_{0}^{\xi} I_{E}(\alpha) \mathrm{d} \alpha\right] \text { and } \\
& K_{L}(\xi)=\exp \left[-\frac{1}{2} \int_{0}^{\xi} I_{L}(\alpha) \mathrm{d} \alpha\right]
\end{aligned}
$$

For the finite pulse tracer input, the regions $0 \leqslant t \leqslant \delta$ and $t>\delta$ must be considcred separately. When $0 \leqslant t \leqslant \delta$, the finite pulse tracer concentration fluctuation response is identical to the step response given by eq. (24). For $t>\delta$, however, the finite pulse tracer concentration fluctuation response is given by

$$
\begin{aligned}
\frac{\sigma^{2}(t)}{C_{f}^{2}}= & q \phi_{i}\left[\int_{t-\delta}^{1} K_{E}(\alpha) f_{i}(\alpha) s(\alpha, \lambda=0) \mathrm{d} \alpha+\int_{\lambda=0}^{\lambda=t} K_{L}(\lambda) \int_{\alpha=\max (t-\lambda-\delta, 0)}^{\alpha=t-\lambda} K_{E}(\alpha) f_{i}(\alpha+\lambda)\right. \\
& \left.\times\left(\frac{\partial s}{\partial \lambda}-\frac{\partial s}{\partial \alpha}\right) \mathrm{d} \alpha \mathrm{d} \lambda\right]+q^{2} \phi_{i}^{2}\left\{\int_{\lambda=0}^{\lambda=t} K_{L}(\lambda) \int_{\alpha=\max (t-\lambda-\delta, 0)}^{\alpha=t-\lambda}\left[1-K_{E}(\alpha)\right] \frac{f_{i}^{2}(\alpha+\lambda)\left(\frac{\partial s}{f(\alpha+\lambda)}-\frac{\partial s}{\partial \alpha}\right) \mathrm{d} \alpha \mathrm{d} \lambda}{f(\alpha)}\right. \\
& +\int_{\alpha=t-\delta}^{\alpha=t}\left[1-K_{E}(\alpha)\right] \frac{f_{i}^{2}(\alpha)}{f(\alpha)} s(\alpha, \lambda=0) \mathrm{d} \alpha-\left[F_{i}(t)-F_{i}(t-\delta)\right]^{2}+\frac{1}{2} \int_{\lambda=0}^{\lambda=t} I_{L}(\lambda) K_{L}(\lambda) \\
& {\left[F_{i}(t)-F_{i}(\max (\lambda, t-\delta))-\int_{\alpha=\max (x-\lambda-\delta, 0)}^{\alpha=t-\lambda} f(\alpha+\lambda) s(\alpha, \lambda) \mathrm{d} \alpha\right]^{2} } \\
& {\left[1-F(\lambda)-\int_{\alpha=0}^{\alpha=\infty} \dot{f}(\alpha+\lambda) s(\alpha, \lambda) \mathrm{d} \alpha\right] }
\end{aligned}
$$


Although these response equations are quite gention because of their complexity. However, they may be simplified to give the response equations for models which are special cases of the master model. For instance, if the master model is restricted by the assumption that $s(\alpha, \lambda)=1-U(\alpha)$, then the master model with mixing by a CRD mechanism reduces to a generalized CRD model similar to that of Treleaven and Tobgy (1972). This same assumption, of course, reduces the master model with mixing by an exchangewith-the-mean mechanism to a generalized IEM model similar to that of Villermaux (1983). Substitution of $1-U(\alpha)$ for $s(\alpha, \lambda)$ in eq. (24) immediately results in the step tracer concentration fluctuation response of either of these models (provided $I=4 \beta$ ) as

$$
\begin{aligned}
\frac{\sigma^{2}(t)}{C_{f}^{2}}= & q \phi_{i} \int_{0}^{t} K(\xi) f_{i}(\xi) \mathrm{d} \xi+\left(q \phi_{i}\right)^{2}\left\{\frac{1}{2} \int_{0}^{t} I(\xi) K(\xi)\right. \\
& \left.\times \frac{\left[W_{i}(t)-W_{i}(\xi)\right]^{2}}{W(\xi)} \mathrm{d} \xi-F_{i}^{2}(t)\right\} .
\end{aligned}
$$

This result is much simpler than eq. (24), yet retains a great deal of generality and is applicable to reactor models of practical significance. Further, if the quadratic in the final integral of eq. (27) is expanded, then eq. (27) may be written as

$$
\begin{aligned}
\frac{\sigma^{2}(t)}{C_{f}^{2}}= & q \phi_{i} \int_{0}^{t} \cdot K(\xi) f_{i}(\xi) \mathrm{d} \xi-\left(q \phi_{i}\right)^{2} F_{i}^{2}(t) \\
& +\frac{\left(q \phi_{i}\right)^{2}}{2}\left[W_{i}^{2}(t) \int_{0}^{t} \frac{I(\xi) K(\xi)}{W(\xi)} \mathrm{d} \xi-2 W_{i}(t)\right. \\
& \left.\times \int_{0}^{t} \frac{I(\xi) K(\xi) W_{i}(\xi)}{W(\xi)} \mathrm{d} \xi+\int_{0}^{t} \frac{I(\xi) K(\xi) W_{i}^{2}(\xi)}{W(\xi)} \mathrm{d} \xi\right]
\end{aligned}
$$

In this form, all of the integrands are independent of time and all of the integrals have time as their upper limit of integration. As shall be seen later, this formularesults.

When the residence time distributions of the two inlet streams are identical (or there is only one inlet stream), eq. (28) may be further simplified. In this case, $W_{i}(\xi) \equiv W(\xi)$, and each of the last three integrals can be integrated by parts to give the result: eral, they are not directly useful in parameter estimation allows for very efficient computation of numerical

way. The first term rises monotonically from zero to the final (steady-state) value of the response, while the second (transient) term rises from zero to some peak value, and decays to its final value of zero.

As another example, consider the case where the master model is restricted by the assumptions that $I_{E}(\alpha)=0$ and $I_{L}(\lambda) \rightarrow \infty$. In this case, the master model reduces to a general three-environment model, analogous to the general two-environment model of Nishimura and Matsubara (1970). Substitution of $I_{E}(\alpha)=0$ and $I_{L}(\lambda) \rightarrow \infty$ into eq. (24) gives the step tracer concentration fluctuation response of this general three-environment model as

$$
\frac{\sigma^{2}(t)}{C_{f}^{2}}=q \phi_{i} G_{i}(t)+\left(q \phi_{i}\right)^{2}\left\{\frac{\left[F_{i}(t)-G_{i}(t)\right]^{2}}{1-G(\infty)}-F_{i}^{2}(t)\right\}
$$

where $G_{i}(t)$ and $G(t)$ are auxiliary integrals defined by

$$
G_{i}(t)=\int_{0}^{t} f_{i}(\alpha) s(\alpha, \lambda=0) \mathrm{d} \alpha
$$

and

$$
G(t)=\int_{0}^{t} f(\alpha) \mathrm{s}(\alpha, \lambda=0) \mathrm{d} \alpha=\sum_{j=1}^{2} \phi_{j} G_{j}(t) .
$$

As with the previous special case, all of the integrals required to evaluate the tracer concentration fluctuation response have time-independent integrands with time as their upper limit of integration. In addition, the environment function appears only as $s(x, \lambda=0)$. This means that (for this case) the tracer concentration fluctuation response is a function of the extent of mixing, but not of the timeliness of mixing. Models exist which differ in when material ultimately exiting from the leaving environment transfers from the entering environment to the leaving environment, but which have the same tracer concentration fluctuation response. Thus, tracer concentration fluctuation response matching has only limited potential for discrimination belween models.

\section{Four-environment model response}

Although it is not a special case of the master model, the four-environment model of Mehta and Tarbell (1983) will be considered because of its practical significance. The four-environment model is defined in the steady state by

$$
\frac{\mathrm{d} \vec{C}_{L, i}(\lambda)}{\mathrm{d} \lambda}=-\mathscr{R}\left[\vec{C}_{L, i}(\lambda)\right]-R \frac{\phi_{i}\left[\overrightarrow{\mathrm{IV}}_{i}(\lambda)-\vec{C}_{L, i}(\lambda) \mathrm{III}(\lambda)\right]+\sum_{j=1}^{2} g_{j}(\lambda)\left[\vec{C}_{L, j}(\lambda)-\vec{C}_{L, i}(\lambda)\right]}{g_{i}(\lambda)}
$$

and

$$
\begin{aligned}
\frac{\sigma^{2}(t)}{C_{f}^{2}}= & q \phi_{i}\left(1-q \phi_{i}\right) \int_{0}^{t} K(\xi) f(\xi) \mathrm{d} \xi \\
& +\left(q \phi_{i}\right)^{2} W^{2}(t) \int_{0}^{t} \frac{K(\xi) f(\xi)}{W^{2}(\xi)} \mathrm{d} \xi .
\end{aligned}
$$

Aside from the obvious compactness of this expression, it also partitions the response in a very natural

$$
\vec{C}_{x}=\sum_{j=1}^{2}\left[\vec{C}_{L, j}(\lambda=0) g_{j}(\lambda=0)+\phi_{j} \overrightarrow{\mathbf{I V}}_{j}(\lambda=0)\right]
$$

where $g_{i}$ is the solution to the differential equation

$$
\frac{\mathrm{d} g_{i}(\lambda)}{\mathrm{d} \lambda}=-R \phi_{i} \operatorname{III}_{i}(\lambda)-R \sum_{j=1}^{2}\left[g_{j}(\lambda)-g_{i}(\lambda)\right]
$$


and the auxiliary integrals III and IV are defined by

$$
\operatorname{III}_{i}(\lambda)=\int_{0}^{\infty} \exp (-R \alpha) f_{i}(\alpha+\lambda) \mathrm{d} \alpha
$$

and

$$
\overrightarrow{I V}_{i}(\lambda)=\int_{0}^{\infty} \vec{C}_{b}\left(\alpha, \vec{C}_{f, i}\right) \exp (-R \alpha) f_{i}(\alpha+\lambda) \mathrm{d} \alpha
$$

and where $\vec{C}_{b, i}$ is the solution to the batch kinetics:

$$
\frac{\mathrm{d} \vec{C}_{b}(\alpha)}{\mathrm{d} \alpha}=\mathscr{R}\left[\vec{C}_{b}(\alpha)\right]
$$

subject to

$$
\vec{C}_{b, i}(0)=\vec{C}_{f, i}
$$

In order to determine the tracer concentration fluctuation response of this model, the model must be formulated for unsteady-state application, and the equation for the mean squared concentration of the reactor effluent must be determined. If the model is extended to accommodate unsteady-state operation, then restricted to the single-component nonreactive case one obtains the leaving environments' equations of change as solved by direct integration. The equation of change for $g_{2}-g_{1}$ was solved by an integrating factor technique. From these, $g_{1}$ and $g_{2}$ were determined separately. Equations of change for $g_{1} C_{L, 1}+g_{2} C_{L, 2}$ and $g_{2} C_{L, 2}-g_{1} C_{L, 1}$ were formulated based on eq. (40). The equations were solved for the cases of step and finite pulse tracer inputs to a single inlet, using direct integration for the former equation and an integrating factor technique for the latter. From these, the first two moments of the efflucnt concentration distribution were evaluated as a function of time. For the case of a step tracer input, the resulting tracer concentration fluctuation response is

$$
\begin{aligned}
\frac{\sigma^{2}(t)}{C_{f}^{2}}= & \left(\frac{1}{2} q \phi_{i}\right)^{2}\left(\frac{\left[F_{i}(t)-\exp (-R t) G_{i}(t)-R H_{i}(t)\right]^{2}}{g_{i}}\right. \\
& \left.+\frac{\left\{F_{i}(t)-[2-\exp (-R t)] G_{i}(t)+R H_{i}(t)\right\}^{2}}{g_{k}}\right) \\
& -\left(q \phi_{i}\right)^{2} F_{i}^{2}(t)+q \phi_{i} G_{i}(t) .
\end{aligned}
$$

where $G_{i}$ is given by eq. (31) with $s(\alpha, \lambda)=\exp (-R \alpha)$, and also

$$
\frac{\partial C_{L, i}(\lambda, T)}{\partial \lambda}=-R \cdot \frac{\phi_{i}\left[\operatorname{IV}_{i}^{*}(\lambda, T)-C_{L, i}(\lambda, T) \operatorname{III}_{i}(\lambda)\right]+\sum_{i=1}^{2} g_{j}(\lambda)\left[C_{L, j}(\lambda, T)-C_{L, i}(\lambda, T)\right]}{g_{i}(\lambda)}
$$

and the equations for the moments of the reactor effuent concentration distribution as

$$
\begin{aligned}
C_{x}(t)= & M_{1, x}(t)=\sum_{j=1}^{2}\left[C_{L, j}(\lambda=0, t) g_{j}(\hat{\lambda}=0)\right. \\
& +\phi_{j}\left[V_{j}^{*}(\lambda=0, T)\right]
\end{aligned}
$$

and

$$
\boldsymbol{M}_{2, \boldsymbol{x}}(t)=\sum_{j=1}^{2}\left\{\left[C_{L, j}(\lambda=0, t)\right]^{2} g_{j}(\lambda=0)\right.
$$

$$
\begin{aligned}
g_{i}= & g_{i}(\lambda=0)=\frac{1}{2}\left\{1-G(\infty)+\phi_{i}\left[G_{i}(\infty)-R H_{i}(\infty)\right]\right. \\
& \left.-\phi_{k}\left[G_{k}(\infty)-R H_{k}(\infty)\right]\right\}
\end{aligned}
$$

and

$$
H_{i}(t)=\int_{0}^{t} \exp (-R \alpha) G_{i}(\alpha) \mathrm{d} \alpha .
$$

For a finite pulse tracer input, the response is identical to the step response for $0 \leqslant t \leqslant \delta$. For $t>\delta$, the tracer concentration fluctuation response becomes

$$
\begin{aligned}
\frac{\sigma^{2}(t)}{C_{f}^{2}}= & \left(\frac{1}{2} q \phi_{i}\right)^{2}\left(\frac{\left\{\left[F_{i}(t)-F_{i}(t-\delta)\right]-\exp (-R t) G_{i}(t)+\exp [-R(t-\delta)] G_{i}(t-\delta)-R\left[H_{i}(t)-H_{i}(t-\delta)\right]\right\}^{2}}{g_{i}}\right. \\
& \left.+\frac{\left\{\left[F_{i}(t)-F_{i}(t-\delta)\right]-[2-\exp (-R t)] G_{i}(t)+[2-\exp (-R(t-\delta))] G_{i}(t-\delta)+R\left[H_{i}(t)-H_{i}(t-\delta)\right]\right\}^{2}}{g_{k}}\right) \\
& -\left(q \phi_{i}\right)^{2}\left[F_{i}(t)-F_{i}(t-\delta)\right]^{2}+q \phi_{i}\left[G_{i}(t)-G_{i}(t-\delta)\right] .
\end{aligned}
$$

$$
\left.+\phi_{j} \int_{0}^{\infty} C_{f, j}^{2}(t-x) \exp (-R \alpha) f_{j}(\alpha) \mathrm{d} \alpha\right\}
$$

with $g_{i}(\lambda)$ as before and

$$
\operatorname{IV}_{i}^{*}(\lambda, t)=\int_{0}^{\infty} C_{f, i}(t-\alpha-\lambda) \exp (-R \alpha) f_{i}(\alpha+\lambda) \mathrm{d} \alpha
$$

To solve these equations, the following procedure was used: the equation of change for $g_{1}+g_{2}$ was

\section{PARAMETER ESTIMATION}

Numerical evaluation of response expressions

Once the tracer concentration fluctuation response has been determined, the question arises of how best to calculate numerical values of the response. Since parameter estimation requires repetitive evaluation of the predicted tracer concentration fluctuation response, it is important that numerical evaluation of the response be performed as efficiency as possible.

Initially, the quantitative description of tracer concentration fluctuation responses was posed in terms of 
partial differential equations, and numerical evaluation of the response appeared to be a difficult, if not intractable problem. In light of the analysis presented here, it is apparent that the quantitative description of tracer concentration fluctuation responses can generally be posed in terms of definite integrals; numerical evaluation of the response is reduced to the much simpler problem of numerical quadrature. In the case of the master model, even this is not an easy task, because several of the integrands in cqs (24) and (26) themselves require evaluation of definite integrals. When the master model is restricted to the generalized CRD case or the generalized three-environment case, however, the response involves only single integrals whose upper limit is the exit time, and whose integrands are independent of time. This property can be exploited to enable more efficient computation of tracer concentration fluctuation responses.

The response can be evaluated by calculating values of $\frac{\sigma^{2}(t)}{C_{f}^{2}}$ at a series of times $t_{1}<t_{2}<\ldots<t_{n}$ to form a table from which the response at any desired time may be interpolated. This in turn requires a table of definite integral values for each integral term in the expression being evaluated. This could be calculated by evaluating the definite integral from 0 to $t_{1}$, then the definite integral from 0 to $t_{2}$, etc., for each term. However, when the integrals have the same integrand, the table may be produced by first evaluating the definite integral from 0 to $t_{1}$, then evaluating the definite integral from $t_{1}$ to $t_{2}$ and adding this to the previous result to get the definite integral from 0 to $t_{2}$, and so on. Compared to performing separate integrations, use of this incremental integration algorithm provides a factor of $\left(\frac{n}{2}\right)$ reduction in the total length of all integration intervals, and a corresponding reduction in the computational effort required to evaluate the tracer concentration fluctuation response.

Thus, parameter estimation by tracer concentration fluctuation response matching requires at each iteration (of error minimization) calculation of a small number of integrals which can be effeciently evaluated using an incremental integration algorithm.

On the other hand, parameter estimation by matching of steady-state reactor performance requires at each iteration a full solution of the reactor model for each data point. For the environment models, each model solution requires the evaluation of three integrals (not all amenable to incremental integration), in addition to the solution of coupled ODE sets for each leaving environment. For the IEM model, each model solution requires the solution of a set of non-linear equations, which at each iteration of the equation solver requires the solution of an ODE and evaluation of a definite integral. For the CRD model, the computational burden is greater still, since each model "solution" requires stochastic reactor simulation.

Such a comparison should make it clear that parameter estimation by tracer concentration fluctuation response matching is far less computationally intensive than parameter estimation by steady-state reactor performance matching.

\section{Implementation of algorithms}

A short demonstration program was written to determine whether the method of parameter estimation by tracer concentration fluctuation response matching is feasible and whether the method can discriminate betwecn competing mixing models. This program performed parameter estimation by adjusting a single model parameter so as to minimize the sum of the squared differences between the predicted and measured response values. For this demonstration, only the two-environment model of $\mathrm{Ng}$ and Rippin (1967) and the (constant parameter) CRD model of Kattan and Adler (1972) were considered, and residence time distributions were represented by a tanks-in-series distribution. A golden section search method was used to find the parameter value minimizing the sum of the squared differences, and a packaged Gauss-Kronod quadrature routine was used to evaluate definite integrals.

To test the ability of the parameter estimation method to discriminate between "correct" and "incorrect" models, simulated experimental data was used, generated by a separate computer program for which the correct reactor model was known. The simulated data was generated by stochastic simulation of an unsteady-state CSTR undergoing mixing by a CRD mechanism. The simulation conditions corresponded to a step tracer test with a feed fraction tracer of 0.8 and a mixing parameter of $I=2$. Eight replicate simulations were performed, each using an exit population of 300 cells and a reactor population of 3750 cells. The first simulated data set is based on a single simulation, while the second simulated data set is based on the averaged results from all eight replicate simulations. This second set exhibits less scatter than the first due to the larger aggregate exit population used in the simulation. I Iowever, both sets of simulated data exhibit a small systematic error (with respect to expected values), arising from the discretization of the residence time distribution.

\section{Discussion of results}

The demonstration program was used to determine parameter values for the two-environment and CRD models which best matched the simulated tracer concentration fluctuation response data. For the first simulated data set, it was found that $I=1.952$ for the CRD model and $R=0.8412$ for the two-environment model provided the best fit. Comparison of the predicted tracer concentration fluctuation response and the simulated response data is shown in Fig. 2 for the CRD model and in Fig. 3 for the two-environment model. Plots of the residual errors as a function of time are shown in Fig. 4 for the CRD model and Fig. 5 for the two-environment model. From visual comparison of Figs 2 and 3, it appears that the CRD model provides a somewhat better fit than the two-environ- 


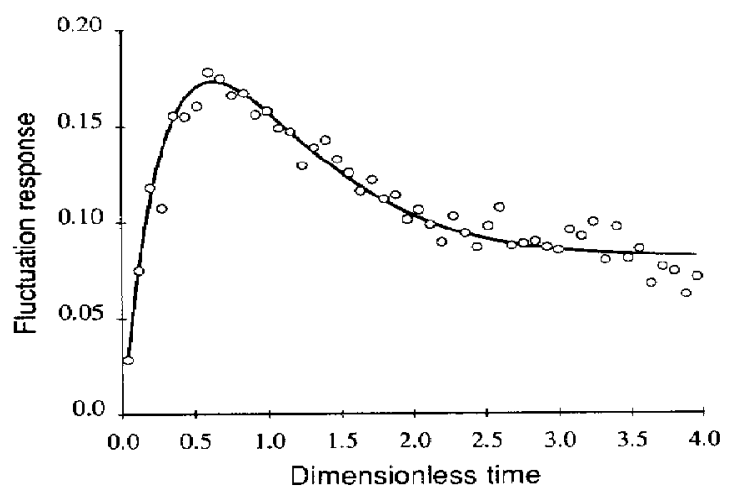

Fig. 2. Comparison between first data set and predicted response for CRD model with $I=1.952$.

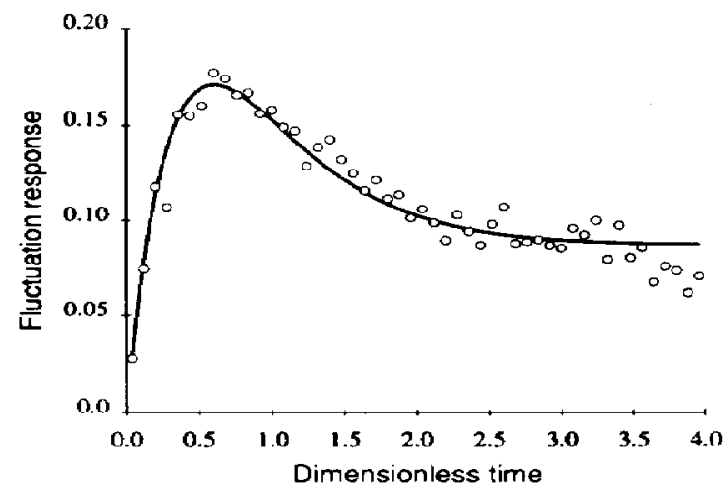

Fig. 3. Comparison between first data set and predicted response for two-environmental model with $K=0.8412$.

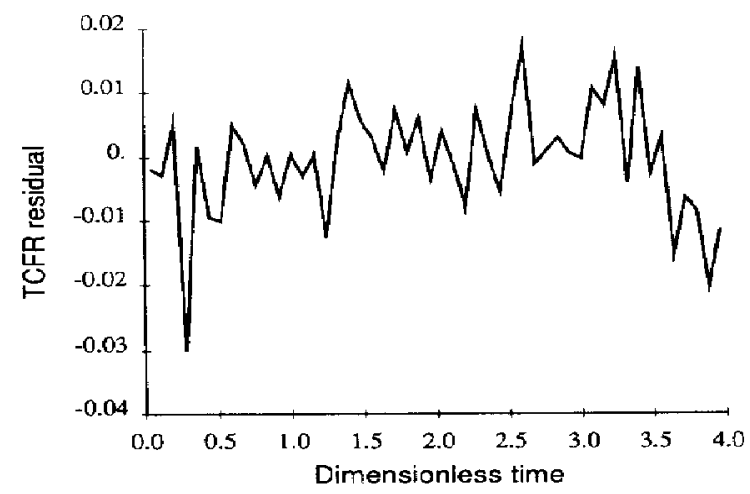

Fig. 4. Residual errors between first data set and CRD model with $I=1.952$.

ment model. The two-environment model appears to predict low values for $1.0 \leqslant t \leqslant 1.75$, and high values for $t>2.5$. The difference in the goodness of fit can be confirmed quantitatively by comparing the sum of the squared residuals. The CRD model gives a minimum error of $3.717 E-03$, while the two-environment model gives a somewhat larger minimum error of $4.532 E-03$.

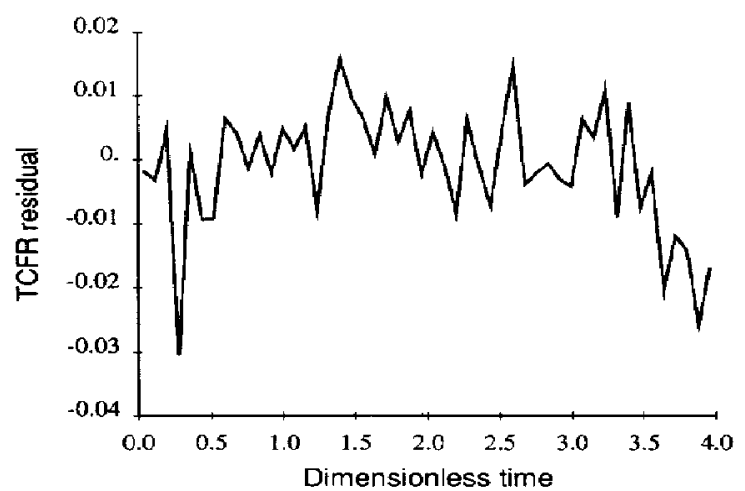

Fig. 5. Residual errors between first data set and twoenvironment model with $R=0.8412$.

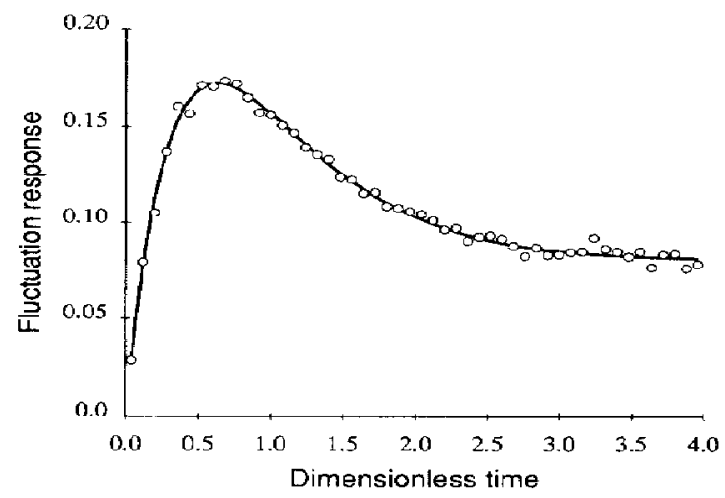

Fig. 6. Comparison between second data set and predicted response for CRD model with $J=1.965$.

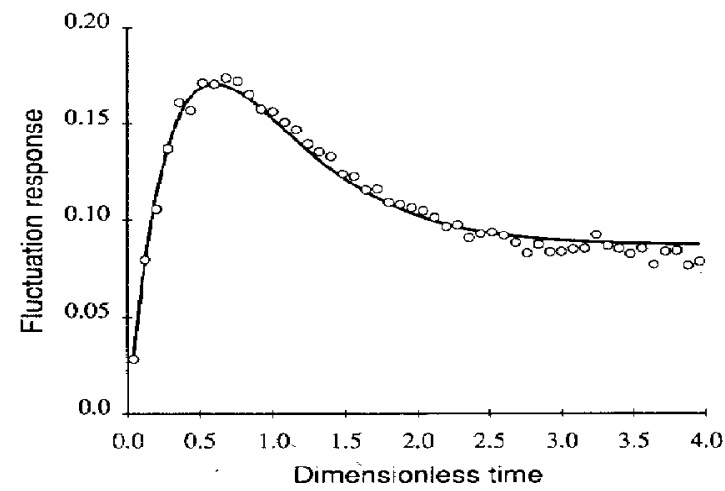

Fig. 7. Comparison between second data set and predicted response for two-environment model with $R=0.8463$.

For the second set of simulated data, parameter estimates of $I=1.965$ for the CRD model and $R$ $=0.8463$ for the two-environment model were calculated. Comparisons of the predicted model tracer concentration fluctuation response and the simulated data are shown in Fig. 6 for the CRD model and in Fig. 7 for the two-environment model. The corresponding residual plots are shown in Figs 8 and 9. A 


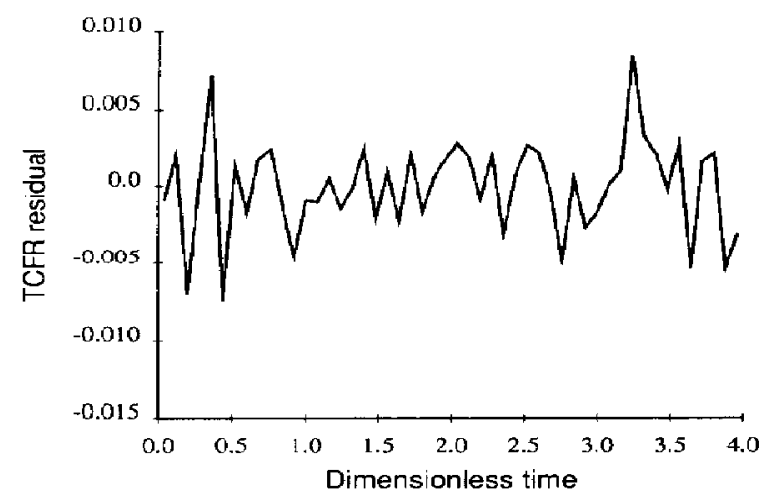

Fig. 8. Residual errors between second data set and CRD model with $I=1.965$.

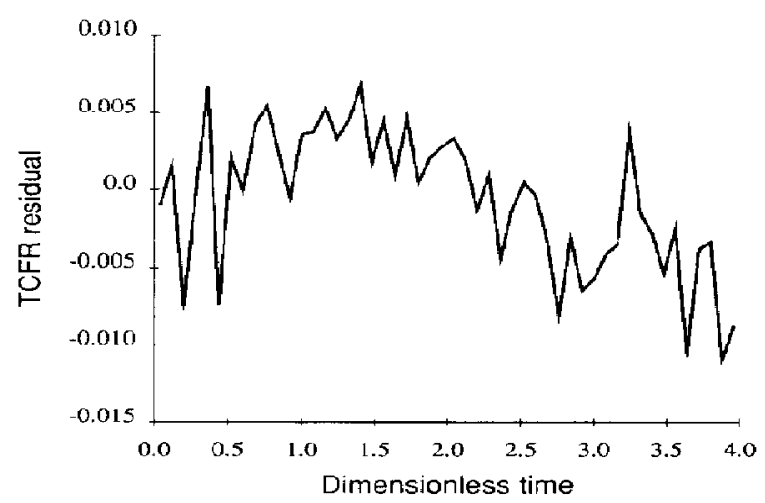

Fig. 9. Residual errors between second data set and twoenvironment model with $R=0.8463$.

comparison of Figs 6 and 7 shows that the CRD model provides a better fit of the simulated data than the two-environment model. As before, the two-environment model tends to underpredict the response at intermediate times and to overpredict the response at large times. However, this trend is more evident with this simulated data set than with the previous one because of the reduced scatter in the second set. The overall curvature of the two-environment residual plot of Fig. 9 shows the trending of the residuals very clearly. The difference in the goodness of fit can once again be confirmed quantitatively by comparing the sum of the squared residuals. The CRD model gives a minimum error of $4.677 E-04$, whereas the two-environment model gives a significantly larger minimum error of $1.033 E-03$.

Another method which can be used for quantitative discrimination between models is statistical analysis of the residuals. If the model adequately represents the data, then the distribution of the residuals between positive and negative values should be random. One measure of the non-randomness of this distribution is the run test (Guttman el al., 1971), in which the number of sequences of consecutive residuals above or below the median is used as the statistic. For the simulated data sets considered here, the number of runs has an expected value of 26 with a standard deviation of approximately 3.5. Analysis of the residuals from the first simulated data set shows that the number of runs is 29 for the fit by the CRD model and 26 for the fit by the two-environment model. Since both of these values fall within an acceptable range, no difference in fit can be detected in this case. For the second simulated data set, it was found that the number of runs was 31 for the fit by the CRD model and $\mathbf{1 5}$ for the fit by the two-environment model. The statistic for the two-environment model is a more than three standard deviations below the expected value. In this case, the hypothesis that the residuals are random in nature can be rejected at the $99 \%$ confidence level, and it can be concluded that there is systematic mismatch between the two-environment model prediction and the second simulated data set.

A second test for discrimination between models is the correlation of the residuals. If the model adequately represents the data, then the residual values should be uncorrelated with the time coordinate. When the residuals were regressed against time for the first simulated data set, the correlation coefficients were 0.071 for the CRD model and -0.222 for the two-environment model. Although the two-environment model shows a stronger correlation, neither correlation coefficient is significantly non-zero in the statistical sense. For the second simulated data set, the residual regression correlation coefficients were 0.076 for the CRD model and -0.552 for the two-environment model. In this case, the hypothesis that residuals are uncorrelated can be rejected even at the $99.99 \%$ confidence level for the two-environment model. On this basis, it can be concluded that there are systematic differences between the two-environment model prediction and the second simulated data set, and that the two-environment model does not fit the simulated data as well as the CRD model.

Comparison of the parameter estimation results using the two simulated data sets shows that the less scattered simulated data set tends to highlight the differences between how well the two mixing models are able to fit the data. It is not surprising that the "better" data allows one to discriminate more clearly between compcting mixing models. The second simulated data set yields a CRD model parameter estimate which differs from the expected value $(2.0)$ by $1.75 \%$, while the first simulated data set yields a parameter estimate that is in error by $2.4 \%$. Although the "better" data set provides the better parameter estimate, the difference in the estimates is small. This suggests that the discrepancy between the parameter estimates and the expected value of 2.0 is largely due to systematic discretization error in the simulation results. This being the case, it appears that the accuracy of the parameter estimates is not adversely affected by the presence of random errors in the simulated data, even for the (more scattered) first data set. For the twoenvironment model, the corresponding expected value of $R$ can be shown to be 1.0 , by matching the 
asymptotic response behavior as is done in the "turbulence analogy" approach to parameter estimation [see, for example, Chaing et al. (1986)]. Using this value leads to the result that the estimated values of $R$ for both simulated data sets are in error by more than $15 \%$. That the "best fits" to the simulated data provide parameter estimates so far from the expected (turbulence analogy) value indicates that perhaps the parameter values predicted by the turbulence analogy approach do not provide the best overall description of reactor behavior. This, of course, is a question which requires further study.

\section{CONCLUSIONS}

A method of micromixing parameter estimation has been proposed based on matching predicted and measured tracer concentration fluctuation responses. Analysis is presented in terms of a master model which conceptually ties together several mixing models. This analysis demonstrates that the tracer concentration fluctuation response can be calculated in terms of definite integrals for a wide variety of Lagrangian mixing models. These predicted responses can be calculated very efficiently for several models of practical significance using an incremental integration algorithm. As a result, parameter estimation calculations using the proposed method are potentially far less computationally intensive than calculations for methods based on steady-state reactor performance matching-

Preliminary numerical tests indicate that the method of tracer concentration fluctuation response matching is able to determine parameter estimates with reasonable accuracy, even when there is significant scatter in the data used for parameter estimation. These tests also indicate that discrimination between competing models is possible in cases where the predicted tracer concentration fluctuation responses for the models differ. Discrimination may be based qualitatively on the goodness of fit of the predicted response to the data, or based quantitatively on statistical discrimination tests.

The proposed tracer concentration fluctuation response matching method shows great promise, and merits additional investigation to confirm its computational superiority to established parameter estimation methods, and to assess the performance of this new parameter estimation method for a variety of real and simulated data sets.

\section{NOTATION}

C concentration, $\mathrm{M} / \mathrm{L}^{3}$

$f(-) \quad$ RTD density function

$F(\cdot) \quad$ RTD cumulative function

$g(\cdot)$ leaving environment volume parameter, defined by eq. (35)

$G(\cdot)$ auxiliary integral defined by eqs (31) and (32)

$H(\cdot)$ auxiliary integral defined by eq. (46)

I( $\cdot$ dimensionless rate parameter for mixing by a CRD mechanism
II( $\cdot$ auxiliary integral defined by eq. (6)

III( $\cdot$ ) auxiliary integral defined by eq. (36)

IV $(\cdot)$ auxiliary integral defined by eq. (37), $M / L^{3}$

$K(-) \quad$ auxiliary integral defined by eq. (25)

$m$ number of chemical species in the reactor

$M(\cdot)$ moment of a concentration distribution

$n \quad$ index of concentration distribution moment or number of data points

$P(\cdot) \quad$ concentration distribution (probability density function)

$q$ fraction of a feed stream containing tracer

$\boldsymbol{R}$ dimensionless mixing parameter for environment models

rate (per residence time) of generation of species due to reaction, $M / L^{3}$

$s(\cdot)$ environment (segregation) function for a model

$t \quad$ current dimensionless time

$T$ dimensionless time at which a given population will exit the reactor

$U(\cdot) \quad$ heaviside unit step function

$W(\cdot) \quad$ reactor washout function, $1-F$

\section{Greek letters}

$\alpha \quad$ age of a given population

$\beta \quad$ dimensionless rate parameter for mixing by an IEM mechanism

$\gamma$ dummy concentration variable, $M / \mathbf{L}^{3}$

$\delta \quad$ duration of pulse feed disturbance

$\lambda$ residual lifetime of a given population

$\xi$ dimensionless dummy age/time parameter

$\sigma(\cdot)$ standard deviation of reactor effluent concentration distribution, $\mathbf{M} / \mathbf{L}^{3}$

$\phi \quad$ fraction of total flow entering a given inlet

\section{Superscripts \\ - refers to a mean value \\ $\rightarrow \quad$ refers to a vector quantity}

\section{Subscripts}

$b \quad$ refers to batch reaction conditions

$E$ refers to entering environment

$f \quad$ refers to feed conditions

$i$ refers to the perturbed inlet stream

$j$ refers to an arbitrary inlet stream

$k$ refers to the unperturbed inlet stream

$L \quad$ refers to the leaving environment

$n \quad$ refers to the number of a moment $M$

$x \quad$ refers to exit conditions

\section{REFERENCES}

Barthole, J. P., Maisonneuve, J., Gence, J. N., David, R., Mathieu, J. and Villermaux, J., 1982, Measurement of mass transfer rates, velocity and concentration fluctuations in an industrial stirred tank. Chem. Engng Fundam. 1, 17-26.

Bennani, A., Gence, J. N. and Mathieu, J., 1985, The influence of a grid-generated turbulence on the development of chemical reactions. A.I.Ch.E. J. 31, 1157-1166.

Call, M. L., 1989, Prediction of micromixing parameters from tracer concentration fluctuations. Ph.D. dissertation, University of Michigan, Ann Arbor, MI.

Chaing, L. J., Mehta, R. V. and Tarbell, J. M., 1986, An 
evaluation of models of mixing and chemical reaction with a turbulence analogy. Chem. Engng Commun. 42, 139-155.

Costa, P. and Trevissoi, C., 1972, Reactions with non-linear kinetics in partially segregated fluids. Chem. Engng Sci. 27, 2041-2054.

Curl, K. L., 1963, Dispersed phase mixing: 1. Theory and effects in simple reactors. A.I.Ch.E. J. 9, 175-181.

Evangelista, J. J., Katz, S. and Shinnar, R., 1969, Scale-up criteria for stirred tank reactors. A.J.Ch.E. J. 15, 843-853.

Gibson, C. H. and Schwarz, W. H., 1963, The universal equilibrium spectra of turbulent velocity and scalar fields. J. Fluid Mech. 16, 365384.

Goto, S. and Matsubara, M., 1975, A generalized twoenvironment model for micromixing in a continuous flow reactor-I. Construction of the model. Chem. Engng Sci. 30, $61-70$.

Guttman, I., Wilks, S. S. and Hunter, J. S., 1971, Introductory Engineering Statistics. Wiley, New York.

Hanley, T. R. and Mischke, R. A., 1978, A mixing model for a continuous flow stirred tank reactor. Ind. Engng Chem. Fundam. 17, 51-58.

Hippler, J. G., Hanley, T. R. and Svihla, C. K., 1985, Micromixing determination by RTD and concentration fluctuation measurement. Seventy-eighth Annual Meeting of the AIChE, Paper $81 \mathrm{~h}$, AIChE, New York.

Kattan, A. and Adler, R. J., 1967, A stochastic mixing model for homogencous, turbulent, tubular reactors. A.I.Ch.E.J. 13, $580-585$.

Kattan, A. and Adler, R. I., 1972, A conceptual framework for mixing in continuous chemical reactors. Chem. Engng Sci. 27, 1013-1028.

Klein, J., David, R. and Villermaux, J., 1980, Interpretation of experimental liquid-phase micromixing phenomena in a continuous stirred reactor with short residence times. Ind. Engng Chem. Fundam. 19, 373-379.

McKelvey, K. N., Yieh, H., Zakanycz, S. and Brodkey, R. S., 1975, Turbulent motion, mixing, and kinetics in a chemical reactor configuration. A.I.Ch.E. J. 21, 1165-1176.

Mehta, R. V. and Tarbell, J. M., 1983, A four-environment model of chemical reaction. Part $\mathrm{I}$-model development. A.I.Ch.E. J. 29, 320-329.

Methot, J. C. and Roy, P. H., 1971, Segregation effects on homogeneous second-order chemical reactions. Chem. Engng Sci. 26, 569-576.

Ng, D. Y. C. and Rippin, D. W. T., 1965, The effect of incomplete mixing on conversion in homogeneous reactors. In Third European Symposium on Chemical Reaction Engineering. Pergamon Press, Oxford.

Nishimura, Y. and Matsubara, M., 1970, Micromixing theory via the two-environment model. Chem. Engng Sci. 25, 1785-1797.

Plasari, E., David, R. and Villermaux, J., 1978, Micromixing phenomena in continuous stirred reactors using a Michaelis-Menten reaction in the liquid phase. ACS Symp. Ser. 65, American Chemical Society, Washington, DC.

Ritchie, B. W., 1980, Simulating the effects of mixing on the performance of unpremixed flow chemical reactors. Can. $J$. chem. Engng 58, 626-633.

Ritchie, B. W. and Tobgy, A. H., 1978, General population balance modelling of unpremixed feedstream chemical reactors: a review. Chem. Engng Commun. 2, 249-264.

Ritchic, B. W. and Tobgy, A. H., 1979, A three-environment micromixing model for chemical reactors with arbitrary separate feedst reams. Chem. Engng $J$. 17, 173-182.

Spielman, L. A. and Levenspiel, O., 1965, A Monte Carlo treatment for reacting and coalescing dispersed phase systems. Chem. Engng Sci. 20, 247-254.

Treleaven, C. R. and Tobgy, A. H., 1972, Monte-Carlo methods of simulating micromixing in chemical reactors. Chem. Engng Sci. 27, 1497-1513.

Valderrama, J. O. and Gordon, A. L., 1979, Mixing effects on homogeneous p-order reactions. A two-parameter model for partial segregation. Chem. Engng Sci. 34, 1097-1103.

Villermaux, J., 1983, Mixing in chemical reactors. ACS Symp. Ser. 226, American Chemical Society, Washington, DC.

Villermaux, J. and Devillon, J. C., 1972, Représentation de la coalescence et de la redispersion des domaines de ségrégation dans un fluide par un modèle d'interaction phénoménologique. In Chemical Reaction Engineering. Proceedings of the Fifth European (Second International) Symposium on Chemical Reaction Engineering. Elsevier, New York.

Villermaux, J. and Zoulalian, A., 1969, État de mélange du fluide dans un réacteur continu-à props d'un modèle de Weinstein et Adler. Chem. Engng Sci. 24, 1513-1517.

Weinstein, H. and Adler, R. J., 1967, Micromixing effects in continuous chemical reactors. Chem. Engng Sci. 22, 65-75.

Zwietering, Th. N., 1984, A backmixing model describing micromixing in single-phase continuous-flow systems. Chem. Engng Sci. 39, 1765-1788. 